\title{
Investigating the Usefulness of European Society of Cardiology Guidelines for Hospitalization in Acute Pericarditis at a Single Tertiary Center
}

\author{
João Ferreira ${ }^{1}$, Mariana Luis ${ }^{2}$, Rui Baptista ${ }^{1}$, Sílvia Monteiro ${ }^{1}$, Lino Gonçalves ${ }^{1}$ \\ 1. Cardiology, Centro Hospitalar e Universitário de Coimbra, Coimbra, PRT 2. Rheumatology, Centro Hospitalar e \\ Universitário de Coimbra, Coimbra, PRT
}

Corresponding author: Mariana Luis, maryanaluys@gmail.com

\section{Abstract \\ Background}

The European Society of Cardiology (ESC) guidelines for the diagnosis and management of pericardial diseases identify predictive factors of poor prognosis and advise either in favor or against hospitalization accordingly. We aim to evaluate the adequacy of hospitalization criteria in a cohort of patients presenting to the emergency department (ED) with acute pericarditis.

\section{Methods}

Retrospective analysis of patients admitted to ED with acute pericarditis, from 2009 to 2019. During ED stay, all patients were evaluated by a cardiologist who decided if the patient was to be discharged or hospitalized. Hospitalized and discharged patients were compared regarding the primary outcome, defined by a composite of: the need for pericardiocentesis and/or cardiac surgery, pericarditis recurrence, and all-cause death. The clinical decision was then counterpoised with ESC guidelines.

\section{Results}

A total of 192 patients were included in the analysis (median age 44.5 years old, $83.3 \%$ male) of which 87 (45.5\%) were hospitalized. A total of $25 \%$ registered the primary outcome, mainly due to acute pericarditis recurrence, occurring in $21.9 \%$. Predictors of recurrence were: glucocorticoid therapy (Odds Ratio $[\mathrm{OR}]=11.93,95 \%$ Confidence Inirtval $[\mathrm{CI}] 3.13-45.5$, $\mathrm{p}<0.001)$, fever at admission (OR=2.67, 95\% CI 1.29-5.49, $\mathrm{p}=0.008)$, immunosuppression $(\mathrm{OR}=4.03,95 \% \mathrm{CI} 1.280-12.659, \mathrm{p}=0.017)$ and increased cardiothoracic index (OR 3.85, CI 95\% 1.67-8.86, $\mathrm{p}=0.002$ ). Regarding hospitalisation/discharge decision, the ESC guidelines were respected in $73.4 \%$ of the cases. However, no significant difference in the primary outcome was noted whether the ESC guidelines were respected or not ( $27.5 \%$ vs. $24.3 \%, \mathrm{p}=0.707)$.

\section{Conclusions}

Review began 01/24/2021 Review ended 02/04/2021 Published 02/07/2021

\section{(c) Copyright 2021}

Ferreira et al. This is an open access article distributed under the terms of the Creative Commons Attribution License CC-BY 4.0., which permits unrestricted use, distribution, and reproduction in any medium, provided the original author and source are credited.
Discrepancy between current guidelines and the clinical decision did not translate into a different outcome.

Categories: Cardiology, Internal Medicine

Keywords: hospitalization, acute pericarditis, prognosis, recurrence, guidelines

\section{Introduction}

Acute pericarditis is a common cause of non-ischemic chest pain in the emergency department (ED) [1]. In most cases, it is a benign and self-limited disease in the setting of a recent viral infection, though it can also be a life-threatening condition or leave permanent complications [2]. It may also be the case that acute pericarditis is only a manifestation of a more severe underlying condition, as a neoplasm or an immunemediated disease $[2-4]$.

Given this heterogeneity around acute pericarditis prognosis, the European Society of Cardiology (ESC) guidelines for the diagnosis and management of pericardial diseases identify predictive factors of poor prognosis and advise either in favor or against hospitalization of acute pericarditis patients according to their presence [5]. According to the ESC guidelines, predictors of poor prognosis can be divided into major risk factors - high fever, subacute course (symptoms over several days without a clear acute onset), evidence of large pericardial effusion (defined as the diastolic diameter of echo-free space over $20 \mathrm{~mm}$ ), cardiac tamponade, failure of response after seven days of non-steroidal anti-inflammatory drugs (NSAIDs), and minor risk factors - myopericarditis, immunodepression, trauma and oral anticoagulant therapy [5]. The presence of one or more risk factors (major or minor) or a suspected underlying etiology (non-viral, nonidiopathic) warrants hospital admission. 
However, due to local factors, the risk of nosocomial infections and the shortage of hospital beds, the ESC guidelines recommendations for admission or discharge may not be followed, also because there is a global perception of the overall good prognosis of acute pericarditis. In this study, our aim was to evaluate the adequacy of the hospitalization criteria suggested by the aforementioned ESC guidelines in a cohort of patients presenting to the ED with acute pericarditis.

\section{Materials And Methods}

We conducted a retrospective, observational cohort study including all patients admitted to ED with a clinical diagnosis of acute pericarditis, between January 2009 and May 2019. The study was conducted at a university tertiary hospital with a large ED (on average, 200,000 episodes in 2017). All patients were evaluated during the ED stay by the cardiologist on duty at the time, who ultimately decided if the patient was to be discharged or hospitalized according to his clinical observation. This observation was then confronted with the ESC guidelines recommendation for admission or discharge of the specific patient clinical setting. Patients aged 17 years or younger, diagnosed during hospitalization and evaluated by a noncardiologist physician during ED stay, were excluded from the study.

We examined demographic and clinical characteristics at ED admission and discharge. Comorbidities were selected in categories of cardiovascular disease based on the International Statistical Classification of Diseases and Related Problems (ICD-10). Abnormal chest radiography was defined as any change visualized in the exam possibly related to acute pericarditis, such as pleural effusion, increased cardiothoracic index and signs of infection or malignancy.

We adopted the classification of pericardial effusion reported by Weitzman et al [6]: a small pericardial effusion (PE) is an echo-free pericardial space (sum of the anterior and posterior effusion) $<10 \mathrm{~mm}$, moderate pericardial effusion is an echo-free space of 10 to $20 \mathrm{~mm}$, and a large pericardial effusion is an echo-free space of $>20 \mathrm{~mm}$. The thickness of pericardial effusion was measured and end-diastole confirmed by the onset of the QRS complex. In the case of circumferential effusions, echo-free space was measured in the parasternal long-axis view. For loculated or asymmetric effusions, the pericardium was evaluated in different planes, including off-axis views, recording the largest site and size of the effusion.

Cardiac troponin I (cTnI) was detected by a high-sensitivity assay. This assay uses cTnI-specific monoclonal antibodies directed to different epitopes being able to recognize multiple modifications in circulation [7]. A positive $\mathrm{cTnI}$ value was defined when the titer was $\geqslant 34 \mathrm{ng} / \mathrm{L}$ for men and $\geqslant 16 \mathrm{ng} / \mathrm{L}$ for women. Antinuclear antibodies (ANA) were measured by indirect immunofluorescence on HEp-2 (human epithelial type 2) cells in line with current guidelines [8]. ANA positivity was defined with a titer of $\geqslant 1: 160$.

Patients were classified according to the: (1) clinician's decision of hospitalization versus nonhospitalization and (2) fulfillment of ESC hospitalization criteria, as shown in Figure 1.

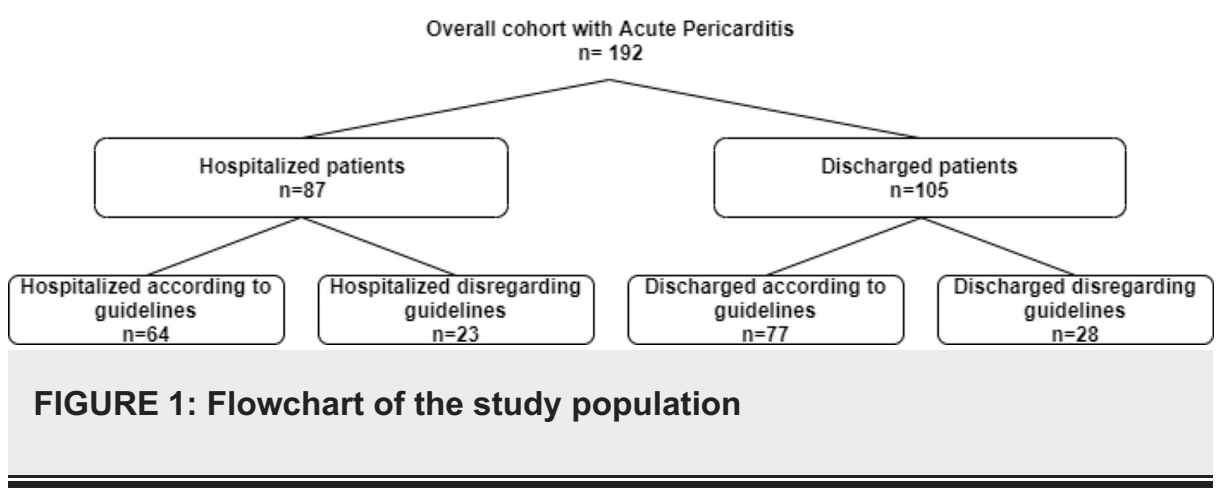

We defined a primary outcome consisting of the composite of (1) need for pericardiocentesis and/or cardiac decompressive surgery, (2) recurrent pericarditis, and (3) all-cause death during the follow-up period (median patient follow-up of 4.0 years \pm interquartile range 5.25 ), and compared the aforementioned groups. Recurrent pericarditis was defined according to ESC guidelines as recurrence of pericarditis after a documented first episode of acute pericarditis and a symptom-free interval of four to six weeks or longer [5].

Quantitative data were expressed as means \pm standard deviation or median with the interquartile range as appropriate and categorical data as frequencies and percentages. Groups were compared using the Student T-test or Mann-Whitney U test for continuous variables and Pearson's $\chi 2$ test for categorical variables. Time to primary outcome distribution was estimated with the Kaplan-Meier method employing the log-rank test. To evaluate potential predictors of unfavorable outcomes, we performed multivariate analysis employing logistic regression with the forward conditional method. Data was analyzed using IBM ${ }^{\circledR}$ SPSS ${ }^{\circledR}$ Statistics, version 22.0 software (IBM Corp., Armonk, NY, USA). 


\section{Cureus}

The authors had full access to data and take full responsibility for its integrity.

\section{Results}

\section{Study population}

We included 192 patients, of which 45.3\% ( $\mathrm{n}=87$ ) were hospitalized (Table 1$)$. The median age was 44.5 years (interquartile range 30.5 ) and $83.3 \%$ were male. Other clinical and demographic features are presented in Table 2. All 192 patients fulfilled the ESC diagnostic criteria for acute pericarditis [5], with pleuritic chest pain being the most common symptom, present in $99.0 \%$ of cases. Typical ECG changes were found in $80.5 \%$ of patients and were more common in males than females $(83.7 \%$ vs. $65.6 \%, p=0.027)$. Transthoracic echocardiography was performed in $81 \%$ of patients $(n=156)$ and PE was present in $50.6 \%$ of cases $(n=79)$, usually mild-moderate (88.9\%), and much more prevalent in females $(82.1 \%$ vs. $43.8 \%$, p $<0.001)$. Idiopathic acute pericarditis was the most prevalent etiology (74.5\%), followed by a recent history of flu-like disease or respiratory tract infection (20.3\%) (Table 3).

\begin{tabular}{|c|c|c|c|c|}
\hline & & \multicolumn{3}{|c|}{ Hospitalized patients (according to clinician) } \\
\hline & & Yes & No & Total \\
\hline \multirow{3}{*}{ Patients fulfilling hospitalization criteria (according to ESC guidelines) } & Yes & 64 & 28 & 92 \\
\hline & No & 23 & 77 & 100 \\
\hline & Total & 87 & 105 & 192 \\
\hline
\end{tabular}

\section{TABLE 1: Study groups}

ESC: European Society of Cardiology.

\begin{tabular}{|c|c|c|c|c|}
\hline Feature & Total $(n=192)$ & Hospitalized patients $(n=87)$ & Not hospitalized patients $(n=105)$ & p-value \\
\hline Age, median (IQR), years & $44.5(30.5)$ & $44.0(30.0)$ & $45(30.0)$ & 0.437 \\
\hline Male sex, n (\%) & $160(83.3)$ & 71 (81.6) & $88(84.6)$ & 0.698 \\
\hline Chest pain, n (\%) & $189(99.0)$ & $85(97.7)$ & $104(100.0)$ & 0.206 \\
\hline Pericardial effusion, n (\%) & $79(50.6)^{*}$ & 48 (61.5) & $32(40.0)$ & 0.007 \\
\hline Large pericardial effusion, $\mathrm{n}(\%)$ & $9(5.7)$ & $9(11.5)$ & $0(0)$ & 0.001 \\
\hline Pericardial rub, n (\%) & $28(14.6)$ & $18(20.7)$ & $10(9.6)$ & 0.040 \\
\hline ECG changes, n (\%) & $148(80.5)$ & 65 (78.3) & $83(82.2)$ & 0.577 \\
\hline Diabetes, n (\%) & $16(8.6)$ & $8(9.8)$ & $8(7.8)$ & 0.793 \\
\hline Arterial hypertension, n (\%) & $48(26.3)$ & $22(26.8)$ & $26(25.2)$ & 0.867 \\
\hline Dyslipidemia, n (\%) & $35(19.4)$ & $17(20.7)$ & $18(17.5)$ & 0.578 \\
\hline Known atrial fibrillation, $\mathrm{n}(\%)$ & $6(3.2)$ & $4(4.9)$ & $2(1.9)$ & 0.408 \\
\hline Known hypothyroidism, n (\%) & $3(1.6)$ & $1(1.2)$ & $2(1.9)$ & 1.000 \\
\hline Chronic kidney disease, n (\%) & $3(1.7)$ & $3(4.1)$ & $0(0)$ & 0.071 \\
\hline
\end{tabular}

\section{TABLE 2: Clinic-demographic features of the study population}

ECG: electrocardiogram; IQR: interquartile range.

*79 patients of the 156 patients that underwent transthoracic echocardiography. 


\section{Cureus}

\begin{tabular}{|c|c|c|c|c|}
\hline Feature & $\begin{array}{l}\text { Total patients } \\
(\mathrm{n}=192)\end{array}$ & $\begin{array}{l}\text { Hospitalized patients } \\
(\mathrm{n}=87)\end{array}$ & $\begin{array}{l}\text { Not hospitalized patients } \\
(n=105)\end{array}$ & $\begin{array}{l}\mathrm{p}- \\
\text { value }\end{array}$ \\
\hline Idiopathic*, (\%) & $142(74.0)$ & 63 (72.4) & $79(76)$ & 0.620 \\
\hline Hypothyroidism, n (\%) & $3(1.6)$ & $1(1.2)$ & $2(1.9)$ & 1.000 \\
\hline HIV infection, n (\%) & $2(1.0)$ & $0(0)$ & $2(1.9)$ & 0.501 \\
\hline Post-cardiac intervention $^{\dagger}, \mathrm{n}(\%)$ & $8(4.2)$ & $5(5.7)$ & $3(2.9)$ & 0.472 \\
\hline Dressler syndrome, n (\%) & $4(2.0)$ & $3(3.4)$ & $1(1.0)$ & 0.332 \\
\hline Chronic kidney disease, $\mathrm{n}(\%)$ & $3(1.6)$ & $3(3.4)$ & $0(0)$ & 0.093 \\
\hline $\begin{array}{l}\text { Recent flu-like/ respiratory tract infection, } \\
n(\%)\end{array}$ & 39 (20.3) & $20(23.0)$ & $19(18.3)$ & 0.473 \\
\hline Neoplasia $^{\mp}, \mathrm{n}(\%)$ & $11(5.7)$ & $5(5.7)$ & $6(5.8)$ & 1.000 \\
\hline Immunomediated disease ${ }^{\mp}, \mathrm{n}(\%)$ & $19(9.9)$ & $6(6.9)$ & $13(12.5)$ & 0.231 \\
\hline
\end{tabular}

\section{TABLE 3: Etiology of acute pericarditis}

*Without a known specific cause/assumed viral cause.

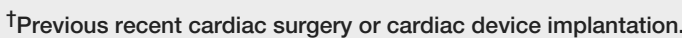

${ }^{\mp}$ Known, diagnosed during hospitalization or diagnosis made $<12$ months after pericarditis episode.

At ED admission, all patients were tested for a complete blood count, C-reactive protein (CRP), highsensitivity cardiac troponin I (hs-cTnI), and ECG. Later, as part of the etiological investigation, 25.5\% ( $\mathrm{n}=49)$ underwent ANA testing, 28.6\% ( $\mathrm{n}=55)$ thyroid function tests and 19.3\% $(\mathrm{n}=37)$ cardiac magnetic resonance (CMR) or chest computed tomography (CT) scan (Table 4). A total of 6.3\% $(n=12)$ of patients underwent emergent coronary angiography for the suspicion of acute myocardial infarction, with no culprit coronary lesions found.

\begin{tabular}{|c|c|c|c|c|}
\hline Feature & $\begin{array}{l}\text { Total patients } \\
(n=192)\end{array}$ & $\begin{array}{l}\text { Hospitalized pateints } \\
(n=87)\end{array}$ & $\begin{array}{l}\text { Not hospitalized patients } \\
(\mathrm{n}=105)\end{array}$ & $\begin{array}{l}\mathrm{p}- \\
\text { value }\end{array}$ \\
\hline CRP, mean (SD), mg/dL & $7.5( \pm 9.0)$ & $11.8( \pm 10.3)$ & $3.7( \pm 5.4)$ & $<0.001$ \\
\hline ESR, mean (SD), mm/1 ${ }^{\text {st }}$ hour & $44.8( \pm 24.5)$ & $48( \pm 25.2)$ & $39.5( \pm 23.4)$ & 0.377 \\
\hline $\begin{array}{l}\text { Leukocyte count, mean (SD), } \\
x 10^{6} / \mathrm{mm}^{3}\end{array}$ & $11.3( \pm 4.6)$ & $12.3( \pm 3.7)$ & $10.6( \pm 5.1)$ & 0.009 \\
\hline Positive hs-cTnl, n (\%) & $35(18.2)$ & $31(36.5)$ & $4(3.8)$ & $<0.001$ \\
\hline Positive ANA, n (\%) & $25(13.0)$ & $12(40.0)$ & $13(68.4)$ & 0.079 \\
\hline Abnormal chest X-ray (\%) & $50(26.0)$ & $33(55.0)$ & $17(23.9)$ & $<0.001$ \\
\hline Performed CT scan (\%) & 26 (13.5) & $12(13.8)$ & 14 (13.5) & 1.000 \\
\hline Performed CMR (\%) & $11(5.7)$ & $8(9.2)$ & $3(2.9)$ & 0.115 \\
\hline
\end{tabular}

\section{TABLE 4: Analytical and imaging tests performed}

ANA: antinuclear antibodies; CMR: cardiac magnetic resonance; CRP: C-reactive protein; ESR: erythrocyte sedimentation rate; hs-cTnl: highsensitivity cardiac troponin I; SD: standard deviation.

Pericardiocentesis was performed in four patients (2.1\%): two patients in the context of cardiac tamponade and two other patients as a diagnostic procedure. Cardiac surgery with the creation of a pericardial window 
NSAIDs were the most prescribed drugs (95.7\%), while combination therapy with NSAIDs and colchicine was used in $55.1 \%$ of patients. Glucocorticoids were prescribed in $7.0 \%$ of patients, of whom $61.5 \%$ had an underlying disease potentially associated with acute pericarditis.

\section{Primary outcome}

The median admission length was 5.0 days (interquartile range 5.0). There were no deaths during ED stay or admission. Five patients (2.6\%) died during the follow-up period for reasons not related to pericarditis or its complications: two deaths related to a malignant neoplasm, one to human immunodeficiency virus (HIV) infection complications, one to severe sepsis, and one to heart failure. The primary outcome occurred in one-quarter of patients $(n=48)$, essentially driven by pericarditis recurrence. Recurrent pericarditis comprised $87.5 \%$ of the primary outcome events. Pericardiocentesis was performed in three cases and two patients required cardiac surgery. Having at least one ESC major risk criteria was predictive of recurrence (OR 3.14, 95\% CI 1.59-6.19, $\mathrm{p}=0.001$ ). The same did not apply for minor criteria (OR 1.47, 95\% CI 0.69-3.14, $\mathrm{p}=0.315$ ). In univariate analysis, predictors of recurrence were: glucocorticoid therapy usage (OR 11.93, 95\% CI 3.13-45.5, $\mathrm{p}<0.001$ ), fever on admission (OR 2.67, 95\% CI 1.29-5.49, $\mathrm{p}=0.008$ ), drug-induced or HIVassociated immunosuppression (OR 4.03, 95\% CI 1.280-12.659, $\mathrm{p}=0.017$ ), and abnormal chest radiography (OR 5.42, CI 95\% 2.33-12.62, p<0.001), particularly increased cardiothoracic index (OR 3.85, CI 95\% 1.67$8.86, \mathrm{p}=0.002)$. Recurrence rate and time to recurrence did not differ between hospitalized and nonhospitalized patients (log-rank $\mathrm{p}=0.932$ ), as shown in Figure 2 .

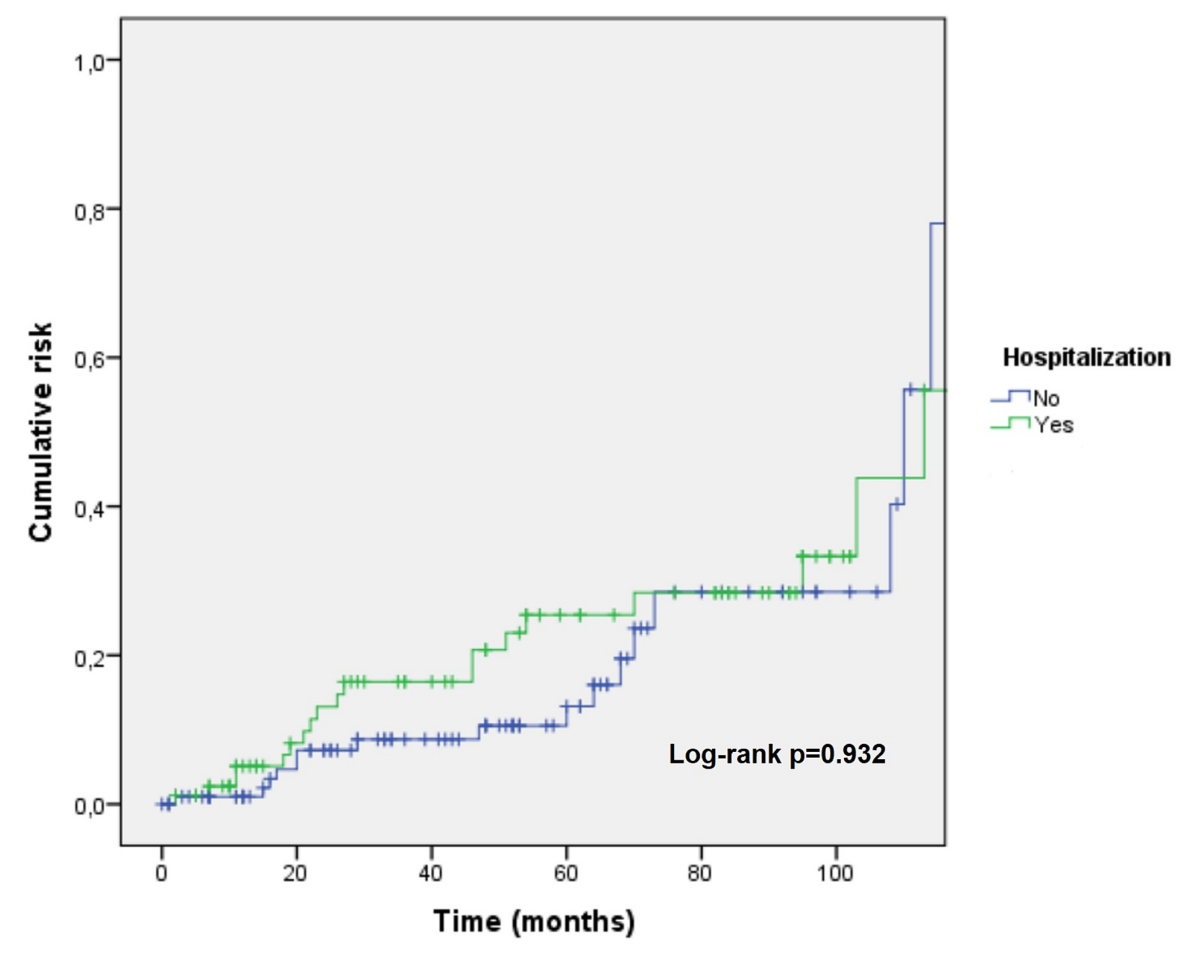

FIGURE 2: Kaplan-Meier curve depicting cumulative risk of recurrence of acute pericarditis between hospitalized and non-hospitalized patients

The use of colchicine became more frequent after 2015 with the publication of the most recent guidelines (79.2 \% vs. $24.1 \%$, p 0 001). Despite not being significantly different, the change in management led to a numerical reduction in the primary outcome ( $16.7 \%$ vs. $24.5 \%, \mathrm{p}=0.213$ ).

\section{ESC-guidelines admission criteria analysis}

Regarding hospitalization/non-hospitalization decision, the ESC guidelines recommendations were followed in the large majority $(73.3 \%, \mathrm{n}=140)$ of the cases (Table 1$)$. Conversely, translated into $26.7 \%(\mathrm{n}=51)$ of patients being admitted without criteria or discharged while having at least major or minor risk factor. Almost half the patients $(47.9 \%, \mathrm{n}=92)$ fulfilled admission criteria and a similar proportion was indeed hospitalized (45.5\%, $\mathrm{n}=87$ ). However, among admitted patients, only $72.4 \%(\mathrm{n}=63)$ fulfilled the admission criteria. On the other hand, $30.8 \%(\mathrm{n}=28)$ patients who fulfilled admission criteria were discharged from the 
ED. Notwithstanding the disregard of ESC guidelines recommendations in this cohort of acute pericarditis patients, we found no significant differences in the primary outcome whether the ESC guidelines were followed or not $(27.5 \%$ vs. $24.3 \%$, p=0.707, respectively). Regarding a single component of the primary outcome, pericarditis recurrence, its incidence was also similar between the two groups (25.5\% vs. $21.4 \%$, $\mathrm{p}=0.561)$.

Regarding the patients discharged from the ED, there was a non-significant numerical reduction in the primary outcome in the group of patients who were correctly discharged following guidelines (14.5\% vs. $28.6 \%, \mathrm{p}=0.150)$.

Finally,, regarding patients ultimately admitted to the hospital, the primary outcome was not different when clinicians followed guidelines criteria for patient hospitalization comparing to non-recommended hospitalizations ( $28.6 \%$ vs. $13.6 \%$, p=0.251).

\section{Discussion}

In this study, we assessed the prognosis and the adequacy of ESC-guidelines defined admission criteria in a cohort of patients presenting to the ED with acute pericarditis.

We found, as described in the literature, that patients with acute pericarditis have an excellent prognosis with rare complications and almost no mortality. However, we still tend towards hospitalization of these patients, even in the total absence of predictors of poor prognosis. Acute phase markers (mainly CRP and leukocyte count), although not considered predictors of poor prognosis [5], were likely to contribute to hospitalization decision, as evidenced by higher levels among hospitalized patients.

Among non-hospitalized patients who fulfilled criteria for hospitalization, the prognosis remains excellent and similar to the ones who were hospitalized. This raises the question of whether some of these patients really need to be hospitalized instead of keeping a follow-up on an outpatient basis.

The clinic-demographic characteristics of our cohort were similar to the previous series. As previously shown, pericarditis has a higher incidence in a middle-aged population, has a male preponderance, a recurrence rate around $15-30 \%$ and a low major complication rate [9-11].

The absence of deaths directly attributed to acute pericarditis is also in line with other studies, as evidenced by a Spanish study where no deaths were registered [12], making it difficult to identify predictors of poor outcome and mortality. Nevertheless, in a Finnish study that included 1,361 admissions for acute pericarditis, increased age and severe coinfection were strong predictors of in-hospital mortality [13]. This seems to imply that, while acute pericarditis has an overall excellent prognosis, a nosocomial infection arising from patient hospitalization is associated with a worse prognosis, suggesting that hospitalization may be harmful in some cases.

Very few studies focus on the decision of hospitalization versus discharge in acute pericarditis patients, although the current ESC guidelines specifically address this issue. One Australian study, including 179 patients with clinic-demographic features similar to our cohort, mentions a $26.8 \%$ hospitalization rate, which is quite low compared to ours [14]. In this study, hospitalization decision was based on the number of risk factors present. Still, no comparison is made regarding the outcome of hospitalized versus discharged patients.

Although the ESC guidelines and the clinical reasoning regarding admission indication were consistent most of the time, in almost a quarter of the cases they differed. Still, this did not translate into a different outcome, raising the question of whether those patients really took benefit from hospitalization.

The answer to this question will depend on whether we, as clinicians, are more focused on the prevention and monitoring of acute pericarditis complications or the etiological investigation of a possible underlying and treatable disease.

The yield of hospital investigation of the etiology of acute pericarditis was low, as we established the diagnosis of idiopathic pericarditis in $74 \%$ of cases. This finding goes in line with other studies in which the underlying etiology was found in about 14 to $22 \%$ of the cases and the vast majority of cases were related to infectious etiology or other unknown causes [11]. The most common specific causes described in published clinical studies with unselected populations are neoplastic (5.1 to 7.0\%), tuberculosis (3.8 to 4.0\%), and autoimmune (1.7 to $7.3 \%$ ). In our study, underlying etiologies are similar to the literature, except for autoimmune cause, which was slightly higher compared to other studies. This could be explained by the low median age of our sample, where autoimmune diseases are more common.

Moreover, if we add other factors as nosocomial infection risk and the limited capacity of hospital beds, we may consider a close follow-up of acute pericarditis patients in an outpatient regime as a plausible alternative to hospitalization, mainly in a population susceptible to in-hospital complications such as the 
immunocompromised and the elderly. Outpatient therapy of low-risk acute pericarditis cases was already tested in other centers, with good efficacy and no serious complications after a mean follow-up of 38 months, with aspirin failure being the main cause for hospitalization of those patients [15].

Recurrent pericarditis was the most common complication, affecting around $20 \%$ of the patients, which is consistent with other series described in the literature [2, 11, 16-22]. However, our follow-up period was longer (with a median follow-up of 48 months) than most studies (18 to 31 months described in the previous series) [9-11].

In general, the predictors of poor prognosis were in accordance with the ones presented in ESC guidelines [5]. In our study, the use of glucocorticoids was the strongest predictor for recurrence. This association has already been described in previous studies [2, 10, 23], especially during glucocorticoid tapering. This finding is also explained by a higher likelihood of choosing glucocorticoid therapy in patients with an underlying immune-mediated disease, which is a risk factor for recurrence and poor prognosis per se [18, 20, 24, 25].

It is important to notice that the current ESC guidelines for the diagnosis and management of pericardial diseases were published in 2015 [5], which conditioned some of our results. For instance, colchicine was only used in nearly half of the cases. This is attributed to the fact that, before 2015, the use of colchicine in acute pericarditis had a class IIa indication and level of evidence B [26]. Only after the COPE trial in 2005 [10] and ICAP trial in 2013 [9], colchicine, in association with NSAIDs, became the therapy of choice. In our cohort, there was a significant increase in the prescription of colchicine after the publication of the most recent guidelines. However, in our cohort, the paradigm shift in treatment led only to a non-significant trend towards the reduction of events. Still, we believe that the lack of significance is explained by our small sample size.

Our study has some limitations: (1) its design as a single-center, retrospective study with a relatively small number of patients is responsible for the low number of follow-up events, apart from recurrence; (2) not all patients underwent echocardiogram at admission in the ED, mainly because these patients had enough clinical data to establish a clinical diagnosis of acute pericarditis; (3) as the decision of different cardiologists may vary, ultimately criteria for hospitalization was not uniform; (4) etiology and outcomes may vary compared to other regions since tuberculosis is a quite common cause of pericarditis in developing countries; and (5) while the median follow-up period in our study was four years, the study population was comprised of patients presenting as late of May 2019, so the patients at the later end may have had significantly less follow up duration.

Our study main strength is the fact it was conducted at a tertiary hospital, which explains the vast variety of etiologies encountered. Regardless of the etiology, all patients had their diagnosis confirmed by a cardiologist. Also, as far as we are aware, this is the first study comparing the outcomes of hospitalized and discharged acute pericarditis patients. However, further prospective and multi-center studies and those which address individual major and minor risk factors are needed to support our conclusions or even update guidelines, which were based on a high level of evidence.

\section{Conclusions}

Discrepancy between current guidelines and clinical reasoning did not translate into different outcomes. Although guidelines should always guide clinical decision-making, local factors and clinical judgment have to be taken into account when it comes to the decision to hospitalize or discharge a patient with acute pericarditis. More data is needed in order to confirm our findings or update guidelines.

\section{Additional Information \\ Disclosures}

Human subjects: Consent was obtained or waived by all participants in this study. Animal subjects: All authors have confirmed that this study did not involve animal subjects or tissue. Conflicts of interest: In compliance with the ICMJE uniform disclosure form, all authors declare the following: Payment/services info: All authors have declared that no financial support was received from any organization for the submitted work. Financial relationships: All authors have declared that they have no financial relationships at present or within the previous three years with any organizations that might have an interest in the submitted work. Other relationships: All authors have declared that there are no other relationships or activities that could appear to have influenced the submitted work.

\section{References}

1. Khandaker M, Espinosa R, Nishimura R, et al.: Pericardial disease: diagnosis and management. Mayo Clin Proc. 2010, 85:572-593. 10.4065/mcp.2010.0046

2. Doctor N, Shah A, Coplan N, Kronzon I: Acute pericarditis. Prog Cardiovasc Dis. 2016, 59:349-359. 10.1016/j.pcad.2016.12.001

3. Gouriet F, Pierre-Yves L, Casalta J, et al.: Etiology of pericarditis in a prospective cohort of 1162 cases . Am J Med. 2015, 128:784.E1-784.E8. 10.1016/j.amjmed.2015.01.040 
4. Søgaard K, Farkas D, Ehrenstein V, Bhaskaran K, Bøtker HE, Sørensen HT: Pericarditis as a marker of occult cancer and a prognostic factor for cancer mortality. Circulation. 2017, 136:996-1006.

10.1161/CIRCULATIONAHA.116.024041

5. Adler Y, Charron P, Imazio M, et al.: 2015 ESC Guidelines for the diagnosis and management of pericardial diseases: the Task Force for the Diagnosis and Management of Pericardial Diseases of the European Society of Cardiology (ESC) endorsed by: the European Association for Cardio-Thoracic Surgery (EACTS). Eur Heart J. 2015, 36:2921-2964. 10.1093/eurheartj/ehv318

6. Weitzman L, Tinker W, Kronzon I, Cohen ML, Glassman E, Spencer FC: The incidence and natural history of pericardial effusion after cardiac surgery: an echocardiographic study. Circulation. 1984, 69:506-511. 10.1161/01.CIR.69.3.506

7. Apple S, Sandoval Y, Jaffe A, Ordonez-Llanos J, for the IFCC Task Force on Clinical Applications of Cardiac Bio-Markers: Cardiac troponin assays: guide to understanding analytical characteristics and their impact on clinical care. Clin Chem. 2017, 63:73-81. 10.1373/clinchem.2016.255109

8. Agmon-Levin N, Damoiseaux J, Kallenberg C, et al.: International recommendations for the assessment of autoantibodies to cellular antigens referred to as anti-nuclear antibodies. Ann Rheum Dis. 2014, 73:17-23. 10.1136/annrheumdis-2013-203863

9. Imazio M, Brucato M, Cemin R, et al.: A randomized trial of colchicine for acute pericarditis . N Engl J Med. 2013, 369:1522-1528. 10.1056/NEJMoa1208536

10. Imazio $\mathrm{M}$, Bobbio $\mathrm{M}$, Cecchi $\mathrm{E}$, et al.: Colchicine in addition to conventional therapy for acute pericarditis: results of the COlchicine for acute PEricarditis (COPE) trial. Circulation. 2005, 112:2012-2016. 10.1161/CIRCULATIONAHA.105.542738

11. Imazio M, Cecchi E, Demichelis B, et al.: Indicators of poor prognosis of acute pericarditis . Circulation. 2007, 115:2739-2744. 10.1161/CIRCULATIONAHA.106.662114

12. Gamaza-Chulián S, León-Jiménez J, Recuerda-Núñez M, Camacho-Freire S, Gutiérrez-Barrios A, VargasMachuca JC: Cardiac troponin-T in acute pericarditis . J Cardiovasc. 2014, 15:68-72. 10.2459/JCM.0b013e3283641161

13. Kytö V, Sipilä J, Rautava P: Clinical profile and influences on outcomes in patients hospitalized for acute pericarditis. Circulation. 2014, 130:1601-1606. 10.1161/CIRCULATIONAHA.114.010376

14. Hooper A, Celenza A: A descriptive analysis of patients with an emergency department diagnosis of acute pericarditis. Emerg Med J. 2013, 30:1003-1008. 10.1136/emermed-2012-201755

15. Imazio M, Demichelis B, Parrini I, et al.: Day-hospital treatment of acute pericarditis: a management program for outpatient therapy. J Am Coll Cardiol. 2004, 43:1042-1046. 10.1016/j.jacc.2003.09.055

16. Imazio M, Gaita F: Acute and recurrent pericarditis . Cardiol Clin. 2017, 35:505-513. 10.1016/j.ccl.2017.07.004

17. Kumar N, Pandey A, Jain P, Garg N: Acute pericarditis-associated hospitalisation in the USA: a nationwide analysis, 2003-2012. Cardiol. 2016, 135:27-35. 10.1159/000445206

18. Farzad A, Schussler J: Acute myopericardial syndromes . Cardiol Clin. 2018, 36:103-114. 10.1016/j.ccl.2017.09.004

19. Meurin P, Tabet J: Colchicine in acute pericarditis: a new standard? . Arch Cardiovasc Dis. 2011, 104:425427. 10.1016/j.acvd.2011.06.002

20. Cremer P, Kumar A, Kontzias A, et al.: Complicated pericarditis: understanding risk factors and pathophysiology to inform imaging and treatment. J Am Coll Cardiol. 2016, 68:2311-2328. 10.1016/j.jacc.2016.07.785

21. Imazio M: Contemporary management of pericardial diseases. Curr Opin Cardiol. 2012, 27:308-317. 10.1097/hco.0b013e3283524fbe

22. Mody P, Bikdeli B, Wang Y, Imazio M, Krumholz HM: Trends in acute pericarditis hospitalisations and outcomes among the elderly in the USA, 1999-2012. Eur Hear J - Qual Care Clin Outcomes. 2018, 4:98-105. 10.1093/ehjqcco/qcx040

23. Mager A, Talmor Y, Chezar Azzerad C, et al.: Does colchicine decrease the rate of recurrence of acute idiopathic pericarditis treated with glucocorticoids? J Cardiol. 2018, 71:409-413. 10.1016/j.jjcc.2017.10.005

24. Imazio M, Spodick D, Brucato A, Trinchero R, Markel G, Adler Y: Diagnostic issues in the clinical management of pericarditis. Int J Clin Pract. 2010, 64:1384-1392. 10.1111/j.1742-1241.2009.02178.x

25. Imazio M, Brucato A, Brucato A, et al.: Antinuclear antibodies in recurrent idiopathic pericarditis: prevalence and clinical significance. Int J Cardiol. 2009, 136:289-293. 10.1016/j.ijcard.2008.05.020

26. Maisch B, Seferović P, Ristić AD, et al.: Guidelines on the diagnosis and management of pericardial diseases: executive summary. The Task Force on the Diagnosis and Management of Pericardial Diseases of the European Society of Cardiology. Eur Heart J. 2004, 25:587-610. 10.1016/j.ehj.2004.02.002 